\title{
INTERACTION OF PLATELET ACTIVATING FACTOR ACETYL HYDROLASE (PAF AH) ENZYME IN Gln281 $\rightarrow$ Arg281 MUTATION TOWARD PAF AND ITS MOLECULAR DYNAMIC
}

\author{
Jayarani Fatimah Putri ${ }^{*}$, Widodo ${ }^{1}$, M. Saifur Rohman ${ }^{2}$ \\ ${ }^{1}$ Biology Master Program,Biology Department, Faculty of Mathematics and Natural Sciences, Brawijaya University, Malang, \\ Indonesia \\ ${ }^{2}$ Department of Cardiology and Vascular Medicine, Faculty of Medicine, Brawijaya University- Saiful Anwar Public Hospital, \\ Malang, Indonesia
}

\begin{abstract}
Platelet Activating Factor Acetyl Hydrolase (PAF AH) or LpPLA2 is a key enzyme in myocardial infarction catalyzes the sn-2 acetyl group of Platelet Activating Factor (PAF) into lyso PAF and acetate as non-potent inflammatory molecules. PAF AH plays a critical role in arterial plaque development of Coronary Artery Disease (CAD). A crystal structure of PAF AH complexes with other ligand and effects of amino acid alteration to protein plasma consequence have also been reported. Here we report on the result of molecular docking and Molecular dynamic (MD) simulation carried out for PAF AH wild type (WT)/PAF and mutant Q281R/PAF complexes. The docking result shows that amino acid residues on active site of Q281 PAF AH mutant have not been recognized on PAF AH. Eelectrostatics and hydrophobic bonds significantly reduced in Q281R than in the wild type. The 7500 ps MD simulation Q281R showed less dynamics than WT but enzymatic machinary of mutant Q281R was not interrupted during MD simulation as well as PAF AH wildtype. These findings clearly indicated the important effect of mutant Q281R in PAF AH recognition to its substrate.
\end{abstract}

Keywords: $P A F A H$, Docking, $M D$

\section{INTRODUCTION}

Cardiovascular disease is the largest cause of morbidity and mortality in Indonesia according to WHO in 2011 [1]. This disease is caused by multiple factors including aging, genetic, and lifestyle factors. Coronary Heart Disease (CHD) is the most common disease in the community [2]. CHD events start from endothelial dys-function in the arteries due to risk factors such as diabetes mellitus, dyslipidemia, smoking, hyper-tension, and obesity. Endothelial dysfunction cause low density lipoprotein (LDL)-cholesterol to be trapped within tunica layer and induce inflmmation facilitated by

\footnotetext{
*Corresponding author: Jayarani Fatimah Putri Biology Master Program, Biology Department, Faculty of Mathematics and Natural Sciences, Brawijaya University, Jl. Veteran Malang, Indonesia, 651445. Email: jayaranidna@gmail.com
}

macrophages. This process induces bulky macrophages form, foam cells and fatty streak which causes narrowing of the lumen of the coronary arteries [3-5].

Protein markers that could indicate the occurrence of endothelial dysfunction is Acetyl-hydro lase Platelet Activating Factor (PAF-AH) [3]. PAF AH or LPPLA2 is $\mathrm{Ca}^{2+}$ independent phospo lipase that degrades platelet activating fac-tor (PAF) as a substrate. The interaction of these two molecules is a form of inflammatory res-ponse to athero sclerosis pathological conditions. PAF-AH is a super family of enzymes that cata-lyze fatty acid in sn-2 position (nucleotide subs-titutions) gliserophospolipid acetyl group into lysophospho lipids and acetate which are mole-cules that are non-potent inflammatory agent. This enzyme is $45 \mathrm{kDa}$ and is secreted by macro-phages into the plasma in the active form [6].

One rare mutation located in adjacent to the highly conserve region and also flanked by active 
site residue, Ser273 and Asp296 is the turn of bases A1001 $\square$ G [7]. This mutation is found in population of Japanese women with cardiovascular disease and clinical condition of essential hypertension. These mutations result in amino acid substitutions of Glycine to Arginine number 281 (Q281R) and also cause loss of enzyme catalytic activity of PAF-AH. At the North American population, this mutation is very common and the highest prevalence of mutations causes partial loss of enzymatic activity of PAF-AH [68]. Since this mutation is located in a prominent position and attacked women with essential hypertension and CAD, therefore, this study was conducted to explore the effect of Q281R mutation using in-silico approach.

\section{MATERIALS AND METHODS}

\section{Protein structure preparation}

Three dimensional crystal structure of wild type human PAF AH was retrieved from RSCB PDB [3D59] comprising 383 amino acid residues [9]. 2D structure and Ramachandran plot of mutant Q281R was done by using JPRED [10] and PROCHECK [11, 12] web server. Homology modeling approach was chosen to construct the 3D structure of Q281R using SWISS MODEL [13, 14]. The pocket site checking was analyzed by CASTpweb server [15]. The structure of PAF as ligand was downloaded from PubChem in pdf file format.

\section{Docking PAF onto PAF AH}

To prepare molecules for docking, all molecules were checked using YASARA to fix all atoms. Two hundred times energy minimization was applied for PAF molecule using Open Babel in Pyrx software. Docking molecule between two types of PAF AH and PAF molecules was performed using AutodockVina software with a default parameter for choosing grid (X:25.000, Y:25.000, Z:25000) and the exhaustiveness de-gree was eight. To analyze and overview the docking result, PyMol and DeepView 4.1.0 were used. Interactions between wildtype and mutant of PAF $\mathrm{AH}$ were checked using Ligandscout [16].

\section{Molecular Dynamics Simulation in Water}

Docking results were subjected to molecular dynamics simulation which was performed using
YASARA software, version 13.2.21 with the AMBER03 force field. All complex protein placed in a box that was $10 \AA$ larger than each side of the protein [9]. Hydrogen atoms were added to counterweigh $\mathrm{pH}$ and $\mathrm{pKa}$ value, when the simulation was applied, the pKa value was higher than the $\mathrm{pH}$ value. Ewald method was used in YASARA simulation to compute $\mathrm{pKa}$ in all molecules used [17]. The complex structures were than minimized using steepest-descent method and followed by simulated annealing. Equalizing to the real cell condition, water solvent, $7.0 \mathrm{pH}$ values in $0.9 \% \mathrm{NaCl}$ for $300 \mathrm{~K}$ temperature for 4000 ps were performed. Intramolecular and intermolecular forces were used for multiple time steps 1.25 and 2.5 fs. Simulation was done using Intel Core2Quad $2.83 \mathrm{Ghz}$, with 2 GB of RAM.

\section{RESULTS AND DISCUSSION}

It is known widely that PAF-AH enzyme plays an important role in cardiovascular disease. This protein has been used as a marker for myocardial infarction [18-20]. Interestingly, these enzymes are involved in two different pathways, inflammatory and non-inflammatory, depending on the substrates, oxLDL or PAF. There is no known mechanism accounting for a drift between proand anti-inflammatory functions associated with PAF-AH. It is believed that what determines the final outcome is dictated by the net change in substrates and products, the environment in which the enzyme functions, and the type of cell that expresses the enzyme.

PAF-AH hydrolysis of PAF produces two molecules that are non-inflammatory, Lyso PAF and acetate. However, their interaction has never been studied before, although the active site of PAF-AH and its interaction with other molecules is clearly described [21]. We analyzed interaction between PAF-AH and PAF using a molecular docking by first obtained a 3D structure through homology modeling. The quality and reliability of structure was checked using PROCHECK based on Ramachandran plot, and most favored region and amino acid were $90 \%$ for all total residues (Figure 1).

3D modeling showed that there were no differences for two dimensional structure bet- 
ween mutant $(\mathrm{Q} 281 \mathrm{R})$ and wild type. This result might be caused due to the replacement of only one amino acid to build Q281R mutant.

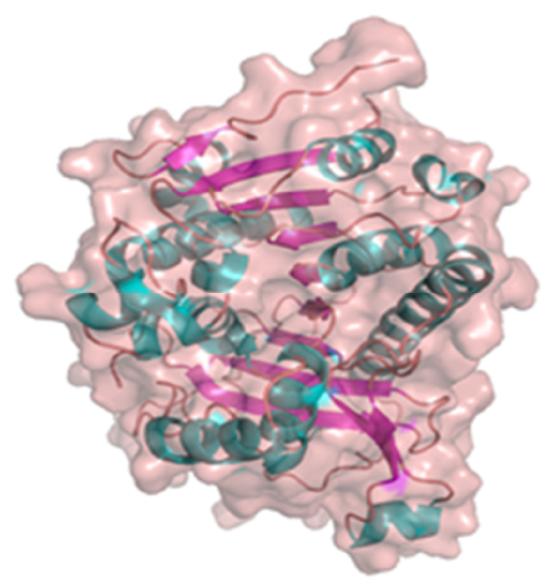

Figure 1. 3D structure of PAF AH
However, we used FoldX from YASARA to check destabilization structure for both structures. Q281R showed many steric clashes (data not shown) and the destabilization value was higher than the wild type structure, with the value of destabilization for Q281R was $-0.57 \mathrm{kcal} / \mathrm{mol}$, much higher than stabilization value of wild type $85.72 \mathrm{kcal} / \mathrm{mol}$. Destabilization structure occurs when there is an increasing number more than $+0.5 \mathrm{kacal} / \mathrm{mol}$ from wild type [22].

To check binding site between PAF AH (wild type and mutant) and PAF, we performed rigid docking. The affinity energy from this process was $-4.0 \mathrm{kcal} / \mathrm{mol}$ to $-2.2 \mathrm{kcal} / \mathrm{mol}$ for wild-type and mutant structures respectively. This value has an important meaning since alteration Glutamine to Arginin may disturb binding process and cause more steric clashes $[23,24]$.
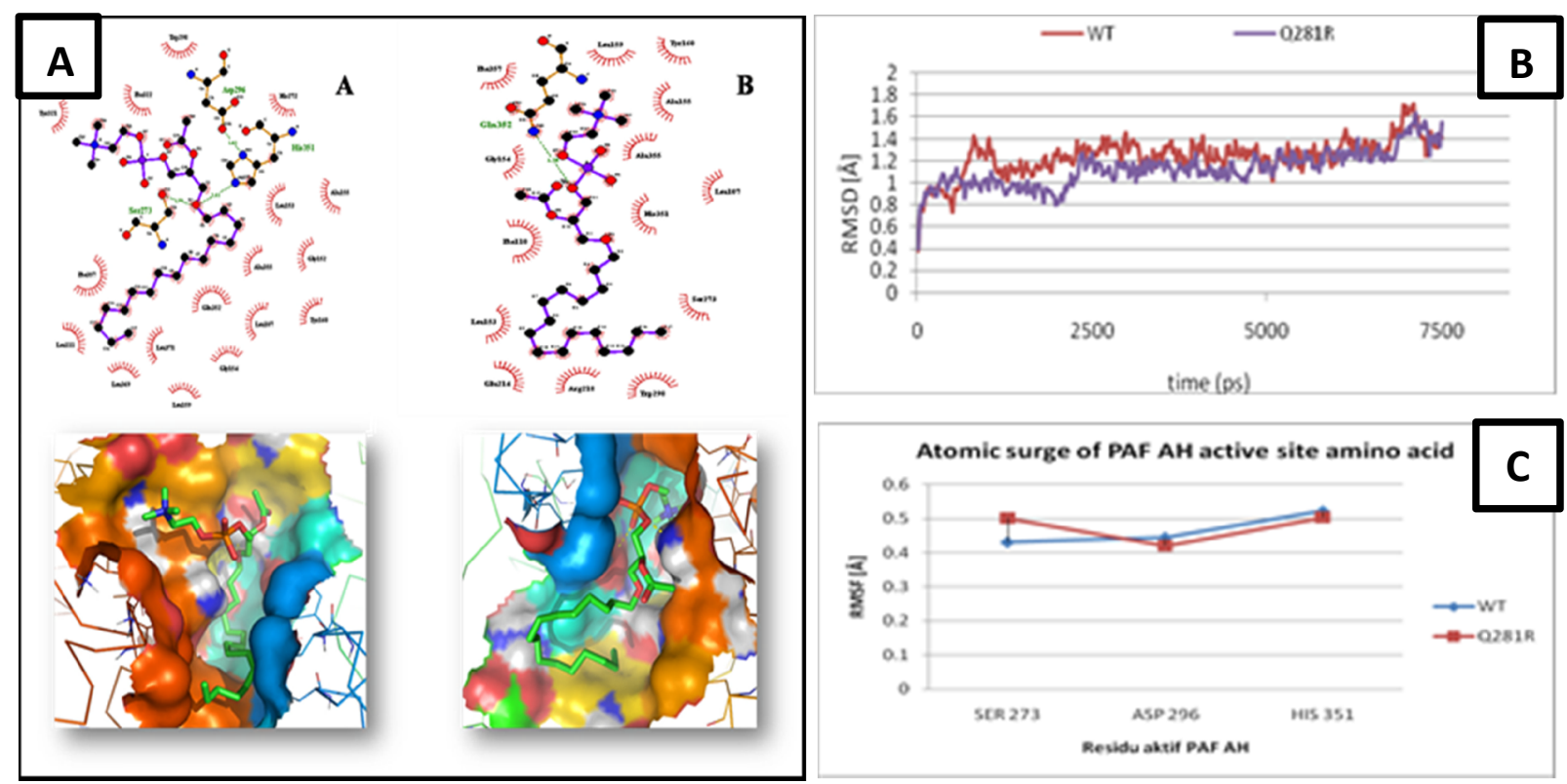

Figure 2. A) Hydrogen and hydrophobic interaction between PAF AH-PAF (above) and PAF position during docking process (below). (A) Wild type, (B) Mutant Q281R. B) C $\alpha$-atomic movement of overall PAF AH residues. C) Active site consisted residues movement.

Using LIGPLUS and LigandScout, all interactions between $\mathrm{PAF} \mathrm{AH}$ (wild type and mutant) and PAF can be mapped (Figure 2A). Wild type docking onto PAF showed three active residues (His351, Ser273, Asp296) of PAF AH enzyme bound to Phospocholine chain of PAF. Otherwise, mutant structure showed no active site residue recognized by PAF as ligand. It seemed that defect on PAF AH structure abolished active site to bind on Phospocholine chain of PAF. Data showed earlier explaining that Glutamin alteration to Arginin may causes enzymatic structural defect that affects enzyme-substrate binding interaction. However, this data still cannot explain how mutation affects enzymatic machinery. Thus, dynamic simulation was performed to figure out this phenomenon. Using water as solvent to adapt for its biological circumstances, we found 
that $\mathrm{C} \alpha \mathrm{RMS}$ atoms movement during simulation for Q281R complex had lower RMSD value that the wild type. The Q281R RMSD value was $1.4 \AA$ and $1.7 \AA$ for wild type. This value means that for all residues of PAF-AH, Q281R is less stable compared to the wild type. No active residues of catalytic sites reached highest peak (Figure 2B) for overall complexes. However, Ser273 residue of active site showed higher atomic movement compared to Asp296 and 351 (Figure 2C).

\section{CONCLUSIONS}

Glutamin $\rightarrow$ Arginin 281 alteration of PAF-AH enzyme causes serious effect for binding recognition with its substrate, PAF. This approach gives new understanding that rare mutation of Q281R PAF-AH may cause serious outcome due to PAF-AH enzyme activity to have been abolished during PAF- PAF-AH recognition. This new finding can be the first evident to make Q281R mutation examination for women CAD patients, especially in developing country.

\section{ACKNOWLEDGMENT}

The researchers would like to thank the funders of the research, Perki Association and Saiful Anwar Public Hospital, Malang.

\section{REFERENCES}

1. WHO (2011) World Health Organization - NCD Country Profiles. www.WHO.int/.

2. George SJ \& Johnson J (2010) Atherosclerosis: Molecular and Cellular Mechanism.Wiley-VCH. Weinheim.

3. Immanuel S \& Tjiptaningrum A (2010) Lipoprotein-Associated Phospholipase A2 (LpPLA2) sebagai Petanda Penyakit Jantung Koroner. Majalah Kedokteran Indonesia. 60(1): 32-39.

4. Loscalzo J (2005) Molecular Mechanism of Atherosclerosis, Taylor \& Francis. United Kingdom.

5. Stafforini DM, Numao T, Tsodikov A, Vaitkus D, Fukuda T, Watanbe N, Fueki N (1999) Deficiency of Platelet Activating Factor Acetylhydrolase is a severity factor for asthma. J Clin Invest. 103: 989-997.

6. Yamada Y \& Yokota M (1998) Roles of Plasma Platelet-Activating Factor Acetylhydrolase in Allergic, Inflammatory, and Atherosclerotic
Diseases, Review Article. Japanese Circulation Journal. 6z:328.

7. Ishihara M, Iwasaki T, Nagano M, Ishii J, Takano M, Kujiraoka T (2004) Functional impairment of two novel mutations detected in LipoproteinAssociated Phospholipase A2 (Lp-PLA2) deficiency patients. J Hum Genet. 49(7): 302.

8. Cornell WD, Cieplak P, Bayly CI, Gould IR, Merz KM Jr, Ferguson DM, Spellmeyer DC, Fox T, Caldwell JW, Kollman PA (1995) A second generation force field for the simulation of proteins, nucleic acids, and organic molecules. J. Am. Chem. Soc. 117: 5179-5197.

9. Cole C, Jonathan DB, Geofrey JB (2008) The Jpred 3 secondary structure prediction server. Nucleid Acid Research. 36: W197-201.

10. Laskowski RA, MacArthur MW, Moss DS, Thornton JS (2003) PROCHECK - a program to check the stereo-chemical quality of protein structures. J. App. Cryst. 26: 283-291.

11. Laskowski RA, Rullmannn J A, MacArthur MW, Kaptein R, Thornton JM (1996) AQUA and PROCHECK-NMR: programs for checking the quality of protein structures solved by NMR. J Biomol NMR. 8: 477-486.

12. Arnold K, Bordoli L, Kopp J, Schwede T (2006) The SWISS-MODEL Workspace: A web-based environment for protein structure homology modelling. Bioinformatics. 22: 195-201.

13. Peitsch MC (1995) Protein modeling by E-mail. Bio/Technology. 13: 658-660.

14. Dundas J, Zheng O, Tseng J, Binkowski A, Turpaz Y, Liang J (2006) CASTp: computed atas of surface topography of proteins with structural and topographical mapping of functionally annotated residues. Nucl. Acids Res. 34: W116W118.

15. Wolber G \& Thierry L (2005) LigandScout: 3-D Pharmacophores Derived from Protein-Bound Ligands and Their Use as Virtual Screening Filters. J.Chem. Inf. Model. 45 (1): 160-169.

16. Krieger E, Nielsen JE, Spronk CAEM, Vriend G (2006) Fast empirical pKa prediction by Ewald summation.J. Mol. Model. 25: 481-486.

17. Bhatti S, Hakeem A, Cilingiroglu M (2010) LpPLA2 as Marker of Cardiovascular Diseases. Curr Atheroscler. 12: 140-144.

18. Ahmed MS, Ji JZ, Meng QH (2011) Lipoproteinassociated phospholipase A2: how effective as a risk marker of cardiovascular disease and as a therapeutic target?. Inflamm Allergy Drug target. 10(4): 236-246.

19. Rosenson RS \& Stafforini DM (2012) Modulation of Oxidation Stress, Inflammation, and Atherosclerosis by Lipoportein-associated Phospolipase A2. Journal of Lipid Res. 53(9): 1767-1782. 
20. Samanta U \& Bahnson BJ (2008) Crystal Structure of Human Plasma Platelet-activating Factor Acetylhydrolase: Structural Implication to Lipoprotein Binding and Catalysis. The Journal of Biological Chemistry. 283(46): 31617-31624.

21. Van Durme J, Delgado J, Stricher F, Serrano L, Schymkowitz J, Rousseau F (2012) A Graphical interface for the FoldX Forcefield. Bioinformatics
27(12): 1711-1712.

22. Payne S, Gayatri P (2005) Geometric Methods in Molecular Docking. Bioinformatics India Journal. 3: 11-12.

23. Mirzaei H, Beglov D, Paschalidis I Ch (2012) Rigid Body Energy Minimization on Manifolds for Molecular Docking. Jornal of Chemical Theory and Computation. dx.i.org/10.1021/ct300272j. 